\title{
ADAPTAÇÃO DO MODELO DE TRANSPORTE PARA UTILIZAR NO PLANEJAMENTO DA PRODUÇÃO CONSIDERANDO MÚLTIPLOS RECURSOS E FAMÍLIAS DE ITENS
}

\author{
Debora Jaensch \\ Programa de Pós-Graduação em Engenharia de Produção UFPR \\ jaensch.debora@gmail.com \\ Neida Maria Patias Volpi \\ Programa de Pós-Graduação em Engenharia de Produção UFPR \\ neida@ufpr.br
}

\begin{abstract}
RESUMO
A área temática deste trabalho é o uso do modelo de transporte como técnica para o planejamento da produção. Um bom plano de produção é aquele que atende as demandas dentro do prazo, respeita a capacidade dos recursos disponíveis e minimiza os custos de produção. Este trabalho busca através da Pesquisa Operacional, adaptar o modelo do transporte ao planejamento da produção, e gerar um aplicativo que sirva de suporte aos tomadores de decisão, fornecendo informações para análise de diferentes cenários do sistema produtivo. Esse aplicativo deve trabalhar com um horizonte de planejamento e custos diferenciados por período e modo de produção, permitir estoque e backlogging, avaliar alterações no planejamento no caso de mudanças na capacidade ou demanda, além de ter fácil utilização, possibilitando assim o gerenciamento fornecendo informações ao usuário.
\end{abstract}

Palavras-chave: Planejamento da Produção; Programação Linear Inteira; Modelo de Transporte 
A área temática deste trabalho é o uso do modelo de transporte como técnica para o planejamento da produção no nível estratégico, onde as decisões são de longo prazo e altos investimentos estão envolvidos.

$\mathrm{O}$ problema de transporte clássico refere-se à distribuição de produtos ao mercado consumidor, de maneira que o custo total seja o menor possível, além de respeitar os limites de oferta e atender às demandas. É possível, através de uma adaptação que será proposta, utilizá-lo no planejamento da produção, servindo como ferramenta de apoio à decisão, e suporte à gestão da produção com foco nas micro e pequenas empresas tanto as que produzem por encomenda quanto as que produzem para estoque.

\section{REVISÃO DE LITERATURA}

O crescimento das indústrias de produção de bens de consumo trouxe consigo a necessidade de um planejamento e maior controle de atividades, em parte devido ao aumento da variedade de produtos e de sua complexidade. Ferramentas que servem de suporte aos planejadores da produção surgiram, tais como o MRP (Materials Requirements Planning), o MRP II (Manufacturing Resources Planning), o ERP (Enterprise Resources Planning), e atualmente o APS (Advanced Planning System) que estão disponíveis no mercado, mas são pouco acessíveis às pequenas empresas.

O planejamento estratégico da produção tem como objetivo adequar os recursos produtivos à demanda esperada, procurando atingir critérios estratégicos de desempenho (custo, qualidade, confiabilidade, pontualidade e flexibilidade). A análise de cenários durante essa etapa auxilia na decisão entre fabricar internamente ou terceirizar, do nível de capacidade com o qual a empresa deve operar, e qual o momento de alteração desta capacidade. Essas decisões estão fortemente ligadas à estratégia de estoques da organização e a flexibilidade de suprimento.

O planejamento agregado da produção é considerado por alguns autores como parte do planejamento estratégico e por outros do planejamento tático. Independente da sua classificação, o planejamento agregado é importante para o planejamento e controle da produção pois de acordo com Gaither e Frazier (2002), desenvolve planos de produção com objetivo de determinar níveis de produção em unidades agregadas ao longo de um horizonte de tempo de médio prazo. Também fazem referência a estoque agregado, utilidades, modificações de instalações e contratos de fornecimentos de materiais.

Neste contexto o modelo clássico de Transportes foi utilizado como inspiração, para que o problema de planejamento da produção fosse tratado através de técnicas da Pesquisa Operacional.

\subsection{MODELO DE TRANSPORTE}

O modelo de transporte é um modelo de programação linear e tem por objetivo minimizar o custo total do transporte necessário para abastecer $n$ centros consumidores (destinos), a partir de $m$ centros fornecedores (origens). Sejam:

- $\quad c_{i j}$ - custo unitário de transporte da origem $i$ para o destino $j$;

- $a_{i}$ - quantidade disponível na origem $i$

- $b_{j}$ - quantidade requerida no destino $j$;

- $x_{i j}$ - quantidade a ser transportada da origem $i$ para o destino $j$. 
A função objetivo (1) minimiza o custo total do transporte, sujeito à restrições de oferta (2) e demanda (3):

$$
\begin{gathered}
\min \sum_{i=1}^{m} \sum_{j=1}^{n} c_{i j} x_{i j} \\
\sum_{j=1}^{n} x_{i j} \leq a_{i}(i=1,2, \ldots m) \\
\sum_{\substack{i=1 \\
m}}^{m} x_{i j}=b_{j}(j=1,2, \ldots, n) \\
x_{i j} \geq 0(i=1,2, \ldots, m) e(j=1,2, \ldots, n)
\end{gathered}
$$

Pizzolato e Gandolpho (2012) afirmam que problemas de programação linear, mesmo que não envolva o transporte de mercadorias, onde os recursos disponíveis e as quantidades necessárias destes recursos são expressos em termos de um só tipo de unidade, podem ser resolvidos pelo algoritmo do transporte, desde que tenham o aspecto do modelo do transporte apresentado acima.

A adaptação deste modelo do transporte para que esse solucione problemas de planejamento da produção é apresentada no decorrer deste trabalho.

\subsection{MODELOS DE PLANEJAMENTO DA PRODUÇÃO}

Arenales et. al (2007) apresentam os principais modelos de planejamento de produção, conhecidos também como modelos para múltiplos itens e recursos, de dimensionamento de lotes (lot sizing) e schedulling. Para todos os modelos são considerados os seguintes dados:

$d_{i t}=$ demanda do item $i$ no período $t$;

$b_{k i}=$ unidades de tempo do recurso $k$ para produzir uma unidade do item $i$;

$C_{k t}=$ capacidade de produção em unidades de tempo da máquina $k$ no período $t$;

$s p_{i}=$ tempo de preparação de máquina para processar o item $i$;

$s_{i}=$ custo de preparação do item $i$;

$h_{i}=$ custo unitário de estoque do item $i$;

$I_{i 0}=$ estoque inicial do item $i$

$n=$ número de itens finais;

$T$ = número de períodos do horizonte de planejamento;

São consideradas também as seguintes variáveis:

$x_{i t}=$ quantidade do item $i$ produzida no período $t$ (tamanho do lote);

$I_{i t}=$ estoque do item $i$ no fim do período $t$

$y_{i t}=\left\{\begin{array}{l}1 \text { se o item i é produzido no período } t \\ 0 \text { caso contrário }\end{array}\right.$

O modelo para múltiplos itens e restrição de capacidade pode ser representado por (4)-(7):

$$
\begin{aligned}
& \min \sum_{i=1}^{n} \sum_{t=1}^{T}\left(s_{i} y_{i t}+h_{i} I_{i t}\right) \\
& I_{i t}=I_{i, t-1}+x_{i t}-d_{i t}, \quad i=1, \ldots, n, \quad t=1, \ldots, T
\end{aligned}
$$




$$
\begin{array}{cl}
\sum_{i=1}^{n}\left(s p_{i} y_{i t}+b_{k i} x_{i t}\right) \leq C_{k t}, & k=1, \ldots, K, \quad t=1, \ldots, T \\
x_{i t} \leq M y_{i t}, & i=1, \ldots, n, \quad t=1, \ldots, T \\
x \in R_{+}^{n T}, \quad I \in R_{+}^{n T}, \quad y \in B^{n T} &
\end{array}
$$

A função objetivo minimiza o custo total de preparação e estoque (4), as restrições (5) correspondem ao balanceamento do estoque de cada item $i$ em cada período $t$, a limitação, em cada período $t$, do tempo total de preparação e produção pela capacidade disponível de cada recurso $k$ está descrito em (6), e $x_{i t}=0$ somente se $y_{i t}=0$ (7). Em casos que a demanda pode ser atendida com atraso, deixa-se a variável de estoque $I$ livre e modifica-se o modelo incluindo uma penalidade $\delta$ por unidade de demanda não atendida no período $t$, onde $I_{i t}^{+}$representa o estoque do item $i$ no fim do período $t$ e $I_{i t}^{-}$a falta (backlogging) do item $i$ no período $t$. Este modelo foi utilizado para adaptar ao de transporte nesta proposta de trabalho.

O trabalho de Erenguc e Tufekci (1988) utiliza o modelo do transporte para solucionar problemas de planejamento agregado, com duas fontes de produção: regular e horas extras, sendo que o modelo permite demanda variada nos diferentes períodos, custos de estoque e atrasos, limites de estoque e atraso. Entretanto, os autores apresentam um planejamento para um único item e um único recurso, o que não o torna prático, pois é difícil encontrar hoje indústrias que tenham recursos específicos para cada item. O modelo apresentado no capitulo 3 generaliza o modelo proposto por Erenguc e Tufekci (1988), para múltiplas famílias de itens e múltiplos recursos compartilhados.

\section{MODELO MATEMÁTICO PROPOSTO}

A proposta de adaptação do modelo do transporte permite flexibilidade no planejamento da produção quanto a prazos, por exemplo, entrega de pedidos atrasados e uso do estoque para atender as demandas, além de permitir a tomada de decisão em função das diferentes estratégias, avaliando o custo de cada cenário proposto. Por exemplo, em uma situação onde a capacidade disponível não atende a demanda do período, vale a pena contratar horas extras, terceirizar a demanda excedente, entregar o pedido em atraso, ou ainda estocar em períodos anteriores?

Para apresentar a adaptação uma analogia é considerada, conforme o QUADRO 1.

\begin{tabular}{|l|l|}
\hline TRANSPORTE & PLANEJAMENTO DA PRODUÇÃO \\
\hline Origens e Destinos & Períodos de Planejamento \\
\hline Custo de Transporte & Custo de Produção \\
\hline Quantidade a ser transportada & Quantidade a ser produzida \\
\hline Demanda do destino $j$ & Demanda do cliente no período $j$ \\
\hline Capacidade de fornecimento na origem $i$ & $\begin{array}{l}\text { Capacidade de produção Normal, com Horas } \\
\text { Extras e Terceirizada no período } i\end{array}$ \\
\hline
\end{tabular}

QUADRO 1 - Analogia entre modelos do transporte e planejamento da produção

FONTE: Os autores (2015) 
As origens e destinos no modelo do transporte são substituídos pelos períodos de planejamento do fornecedor e do cliente, os custos de transporte pelos custos de produção, a quantidade $x_{i j}$ a ser transportada da origem $i$ ao destino $j$ é substituída pela quantidade $x_{i j}$ produzida no período $i$, entregue no período $j$.

A demanda de cada destino passa a ser a demanda de cada período, e por fim a capacidade de cada origem ou fornecedor é substituída pela capacidade de produção disponível em cada período, sendo essa capacidade dividida em 3 modos de produção: capacidade normal $\mathrm{N}$, de horas extras $\mathrm{H}$ e terceirização $\mathrm{T}$. Estas informações são facilmente visualizadas no QUADRO 2, considerando um único tipo de item de produção.

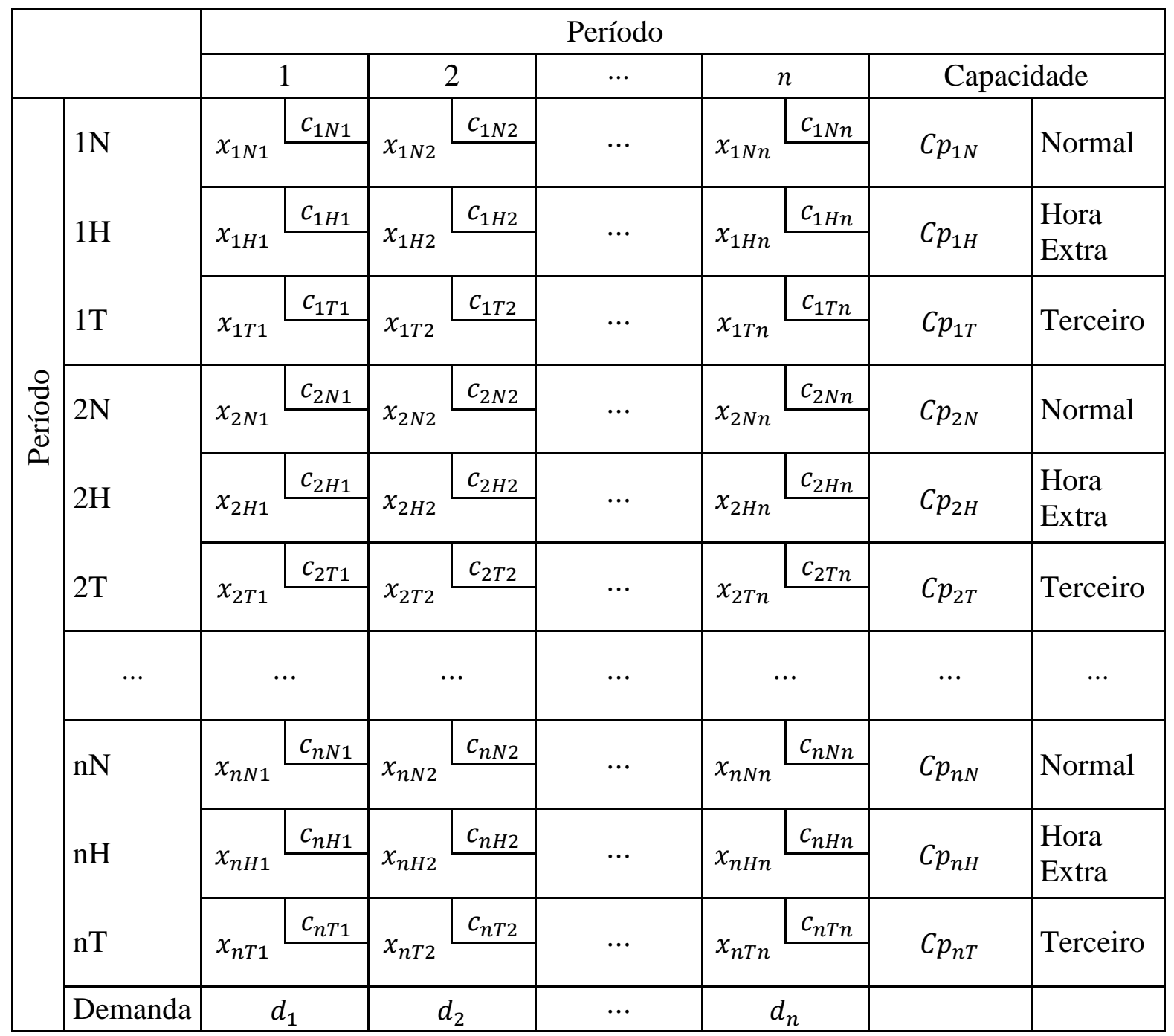

QUADRO 2 - Quadro de planejamento da produção para único tipo de item FONTE: Os autores (2015)

As variáveis $x_{i j}$, com $i=1 N, 1 H, 1 T, \ldots, n N, n H, n T$ e $j=1, \ldots, n$, na adaptação do modelo para planejamento da produção devem ser interpretadas da seguinte forma (QUADRO 3): 


\begin{tabular}{|c|l|}
\hline QUANDO & ENTÃO \\
\hline$i<j$ & $\begin{array}{l}\text { Os itens } x_{i j} \text { produzidos no período } i \text { são estocados e entregues no } \\
\text { período } j, \text { independentes do modo de produção }\end{array}$ \\
\hline$i=j$ & Os itens $x_{i j}$ produzidos no período $i$ são entregues no mesmo período $i$ \\
\hline$i>j$ & $\begin{array}{l}\text { Os itens } x_{i j} \text { produzidos no período } i \text { são entregues com atraso no período } \\
i \text { os pedidos do período } j\end{array}$ \\
\hline
\end{tabular}

QUADRO 3 - Interpretação das variáveis $x_{i j}$

FONTE: Os autores (2015)

A primeira adaptação do modelo do transporte para planejamento da produção, chamado de Modelo I, foi feita para um único item e um único recurso e segue abaixo:

Sejam os dados:

- $\quad c_{i w j}$ - custo unitário de produção no período $i$ e modo de produção $w$ para entrega no período $j$;

- $c e_{i w j}$ - custo unitário acumulado de estoque dos produtos produzidos no período $i$ e modo de produção $w$ e entregues no período $j, i<j$;

- $c b_{i w j}$ - custo unitário acumulado de atraso dos produtos produzidos no período $i$ e modo de produção $w$ e entregues no período $i$ para atender com atraso a demanda do período $j, i>j$;

- $\quad c a p_{i w}$ - capacidade disponível no período $i$ e modo de produção $w$ em unidades de itens;

- $\operatorname{dem}_{j}$ - demanda requerida no período $j$.

Supondo que $x_{i w j}$ represente a quantidade a ser produzida no período $i$ e modo de produção $w$ (normal, hora extra ou terceiro) e entregue no período $j$, conforme interpretação do QUADRO 3, então a função objetivo (8) consiste em minimizar o custo total de produção:

\section{MODELO I}

$$
\begin{gathered}
\min \sum_{i=1}^{n} \sum_{w=1}^{3} \sum_{j=i} c_{i w j} x_{i w j}+\sum_{i=1}^{n} \sum_{w=1}^{3} \sum_{j>i}\left(c_{i w j}+c e_{i w j}\right) x_{i w j} \\
+\sum_{i=1}^{n} \sum_{w=1}^{3} \sum_{j<i}\left(c_{i w j}+c b_{i w j}\right) x_{i w j}
\end{gathered}
$$

Sujeito às restrições:

$$
\begin{gathered}
\sum_{j=1}^{n} x_{i w j} \leq \operatorname{cap}_{i w}(i=1,2, \ldots n ; w=1,2,3) \\
\sum_{\substack{i=1 \\
\sum_{w=1}}}^{3} x_{i w j} \geq \operatorname{dem}_{j}(j=1,2, \ldots, n) \\
x_{i w j} \in Z^{+}(i=1,2, \ldots, n ; w=1,2,3 ; j=1,2, \ldots, n)
\end{gathered}
$$

As $3 n$ restrições, uma de cada período e modo de produção, garantem que a quantidade produzida no período $i$ e modo de produção $w$ tem que ser menor ou igual à capacidade $\operatorname{cap}_{i w}$ disponível naquele período e modo de produção (9), as $n$ restrições de demanda, uma de cada período de entrega, garantem que a quantidade entregue em cada período $j$ tem de ser igual à demanda ${d e m_{j}}_{j}$ requerida(10). 
O modelo I gera como resultado um quadro com o formato apresentado QUADRO 4, onde a diagonal em cinza claro representa as quantidades produzidas e entregues no mesmo período, o triângulo superior direito em branco representa quantidades em estoque e o triângulo inferior esquerdo em cinza escuro representa o backlogging (atraso na entrega).

O modelo I considera apenas um item e um recurso e este trabalho pretende ampliá-lo para vários itens e vários recursos. Essa alteração exige o gerenciamento da capacidade, pois um recurso pode ser utilizado para produção de mais de um item, ou seja, pode ser compartilhado, e o tempo de processamento pode variar de acordo com o item, sendo assim a capacidade não pode mais ser expressa em unidades, pois um recurso pode fazer diferentes quantidades de cada item. No novo modelo (Modelo II) a capacidade será expressa em unidade de tempo (horas, minutos ou segundos), para os modos de produção normal e horas extras, para o modo terceiro continua sendo expressa em unidades de item.

\begin{tabular}{|c|c|c|c|c|}
\cline { 2 - 5 } \multicolumn{1}{c|}{} & 1 & 2 & $\ldots$ & $n$ \\
\hline $1 \mathrm{~N}$ & & & & \\
\hline $1 \mathrm{H}$ & & & & \\
\hline $1 \mathrm{~T}$ & & & & \\
\hline $2 \mathrm{~N}$ & & & & \\
\hline $2 \mathrm{H}$ & & & & \\
\hline $2 \mathrm{~T}$ & & & & \\
\hline$\cdots$ & $\ldots$ & $\ldots$ & $\ldots$ & $\cdots$ \\
\hline$n \mathrm{~N}$ & & & & \\
\hline$n \mathrm{H}$ & & & & \\
\hline$n \mathrm{~T}$ & & & & \\
\hline
\end{tabular}

QUADRO 4 - Apresentação da matriz de resultados do Modelo I

FONTE: Os autores (2015).

Para inclusão de novas famílias de itens $f=1,2, \ldots, F$ os períodos foram subdivididos, criando assim novas colunas, conforme (QUADRO 5).

\begin{tabular}{|c|c|c|c|c|c|c|c|c|c|c|c|c|c|}
\cline { 2 - 13 } \multicolumn{1}{c|}{} & \multicolumn{9}{c|}{1} & \multicolumn{7}{c|}{2} & $\cdots$ & \multicolumn{5}{c|}{$n$} \\
\hline & 1 & 2 & $\cdots$ & $\mathrm{F}$ & 1 & 2 & $\cdots$ & $\mathrm{F}$ & $\cdots$ & 1 & 2 & $\cdots$ & $\mathrm{F}$ \\
\hline $1 \mathrm{~N}$ & $x_{1 N 11}$ & $x_{1 N 21}$ & $\cdots$ & $x_{1 N F 1}$ & $x_{1 N 12}$ & $x_{1 N 22}$ & $\cdots$ & $x_{1 N F 2}$ & $\cdots$ & $x_{1 N 1 n}$ & $x_{1 N 2 n}$ & $\cdots$ & $x_{1 N F n}$ \\
\hline $1 \mathrm{H}$ & $x_{1 H 11}$ & $x_{1 H 21}$ & $\cdots$ & $x_{1 H F 1}$ & $x_{1 H 12}$ & $x_{1 H 22}$ & $\cdots$ & $x_{1 H F 2}$ & $\cdots$ & $x_{1 H 1 n}$ & $x_{1 H 2 n}$ & $\cdots$ & $x_{1 H F n}$ \\
\hline $1 \mathrm{~T}$ & $x_{1 T 11}$ & $x_{1 T 21}$ & $\cdots$ & $x_{1 T F 1}$ & $x_{1 T 12}$ & $x_{1 T 22}$ & $\cdots$ & $x_{1 T F 2}$ & $\cdots$ & $x_{1 T 1 n}$ & $x_{1 T 2 n}$ & $\cdots$ & $x_{1 T F n}$ \\
\hline $2 \mathrm{~N}$ & $x_{2 N 11}$ & $x_{2 N 21}$ & $\cdots$ & $x_{2 N F 1}$ & $x_{2 N 12}$ & $x_{2 N 22}$ & $\cdots$ & $x_{2 N F 2}$ & $\cdots$ & $x_{2 N 1 n}$ & $x_{2 N 2 n}$ & $\cdots$ & $x_{2 N F n}$ \\
\hline $2 \mathrm{H}$ & $x_{2 H 11}$ & $x_{2 H 21}$ & $\cdots$ & $x_{2 H F 1}$ & $x_{2 H 12}$ & $x_{2 H 22}$ & $\cdots$ & $x_{2 H F 2}$ & $\cdots$ & $x_{2 H 1 n}$ & $x_{2 H 2 n}$ & $\cdots$ & $x_{2 H F n}$ \\
\hline $2 \mathrm{~T}$ & $x_{2 T 11}$ & $x_{2 T 21}$ & $\cdots$ & $x_{2 T F 1}$ & $x_{2 T 12}$ & $x_{2 T 22}$ & $\cdots$ & $x_{2 T F 2}$ & $\cdots$ & $x_{2 T 1 n}$ & $x_{2 T 2 n}$ & $\cdots$ & $x_{2 T F n}$ \\
\hline$\cdots$ & $\cdots$ & $\cdots$ & $\cdots$ & $\cdots$ & $\cdots$ & $\cdots$ & $\cdots$ & $\cdots$ & $\cdots$ & $\cdots$ & $\cdots$ & $\cdots$ & $\cdots$ \\
\hline$n \mathrm{~N}$ & $x_{n N 11}$ & $x_{n N 21}$ & $\cdots$ & $x_{n N F 1}$ & $x_{n N 12}$ & $x_{n N 22}$ & $\cdots$ & $x_{n N F 2}$ & $\cdots$ & $x_{n N 1 n}$ & $x_{n N 2 n}$ & $\cdots$ & $x_{n N F n}$ \\
\hline$n \mathrm{H}$ & $x_{n H 11}$ & $x_{n H 21}$ & $\cdots$ & $x_{n H F 1}$ & $x_{n H 12}$ & $x_{n H 22}$ & $\cdots$ & $x_{n H F 2}$ & $\cdots$ & $x_{n H 1 n}$ & $x_{n H 2 n}$ & $\cdots$ & $x_{n H F n}$ \\
\hline$n \mathrm{~T}$ & $x_{n T 11}$ & $x_{n T 21}$ & $\cdots$ & $x_{n T F 1}$ & $x_{n T 12}$ & $x_{n T 22}$ & $\cdots$ & $x_{n T F 2}$ & $\cdots$ & $x_{n T 1 n}$ & $x_{n T 2 n}$ & $\cdots$ & $x_{n T F n}$ \\
\hline
\end{tabular}

QUADRO 5 - Quadro de planejamento da produção para $\mathrm{F}$ itens e vários recursos

FONTE: Os autores (2015). 
Ao incluir novos itens e recursos, houve a necessidade de novas informações através de uma matriz chamada de uso (QUADRO 6). Esse quadro relaciona quantas horas de cada recurso é necessária para produzir cada item de cada família.

O Modelo II fornece uma nova interpretação das variáveis:

- $\quad c_{i w f j}$ - custo unitário de produção no período $i$ e modo de produção $w$ da família de itens $f$ para entrega no período $j$;

- $c e_{i w f j}$ - custo unitário de estoque dos produtos da família de itens $f$ produzidos no período $i$ e modo de produção $w$ e entregues no período $j, i<j$;

- $c b_{i w f j}$ - custo unitário de atraso dos produtos da família de itens $f$ produzidos no período $i$ e modo de produção $w$ e entregues no período $i$ para atender com atraso a demanda do período $j, i>j$;

- $\quad c a p_{i w^{-}}^{k}$ capacidade disponível no período $i$ e modo de produção $w$ para o recurso $k$ em unidades de tempo para os modos de produção normal e horas extras e unidades de item para o modo de terceirização;

- $\operatorname{dem}_{f j}$ - demanda requerida da família de itens $f$ no período $j$ em unidades de itens;

- $\quad x_{i w f j}$ - quantidade a ser produzida da família de itens $f$ no período $i$ e modo de produção $w$ e entregue no período $j$;

- $u_{i f j}^{k}$ - tempo que cada família de item $f$ no período $j$ utiliza do recurso $k$ para ser produzida no período $i$;

A função objetivo (11) consiste em minimizar o custo total de produção:

\section{MODELO II}

$$
\begin{aligned}
\min \sum_{i=1}^{n} \sum_{w=1}^{3} \sum_{f=1}^{F} & \sum_{j=i} c_{i w f j} x_{i w f j}+\sum_{i=1}^{n} \sum_{w=1}^{3} \sum_{f=1}^{F} \sum_{j>i}\left(c_{i w f j}+c e_{i w f j}\right) x_{i w f j} \\
& +\sum_{i=1}^{n} \sum_{w=1}^{3} \sum_{f=1}^{F} \sum_{j<i}\left(c_{i w j}+c b_{i w j}\right) x_{i w j}
\end{aligned}
$$

Sujeito às restrições:

$$
\begin{gathered}
\sum_{j=1}^{n} \sum_{f=1}^{F} u_{i f j}^{k} x_{i w f j} \leq \operatorname{cap}_{i w}^{k}(i=1,2, \ldots n ; w=1,2,3) \\
\sum_{i=1}^{n} \sum_{w=1}^{3} x_{i w f j} \geq \operatorname{dem}_{f j}(f=1,2, \ldots, F ; j=1,2, \ldots, n) \\
(i=1,2, \ldots, n ; w=1,2,3 ; f=1,2, \ldots, F ; j=1,2, \ldots, n)
\end{gathered}
$$

A restrição (12) relaciona as capacidades às famílias de itens de acordo com quanto cada unidade de cada família de item $f$ utiliza da capacidade de determinado recurso $k$ para ser produzida. São definidos uma matriz $u$ e um vetor cap para cada recurso $k$. A restrição (13) garante que a quantidade entregue em unidades de itens em cada período $j$ tem que atender a demanda $\operatorname{dem}_{f j}$ requerida para aquele período.

Uma das vantagens da adaptação do modelo de transporte é a maneira como a solução pode ser apresentada ao usuário, na forma de quadro, informações compactas e visualmente claras em termos do que é atendido em tempo, o que fica atrasado e o que é 
estocado, de cada item e período considerado no planejamento. Outra vantagem é usufruir de toda a teoria e algoritmos propostos que o modelo de transporte, devido a sua importância, têm disponível.

\section{$4 \quad$ APLICAÇÃO DO MODELO II}

Para mostrar uma aplicação do modelo II foram utilizados dados fictícios de forma a aparecerem condições para estoque e backlogging ao mesmo tempo.

Um aplicativo foi desenvolvido, de fácil utilização, voltado para pequenas empresas, sem nenhum processo formal de planejamento com base neste modelo II. $\mathrm{O}$ aplicativo tem como característica trabalhar com um horizonte de planejamento, custos diferenciados por período e modo de produção, permitir ou não estoque e/ou backlogging, avaliar alterações no planejamento em casos de alterações da demanda ou capacidade e possibilitar o gerenciamento fornecendo informações ao usuário. Os softwares Microsoft Excel e LINGO foram usados; o Excel como entrada e saída de dados e o LINGO como software de otimização. A automatização no Excel foi feita através do desenvolvedor Visual Basic.

Quanto à entrada de dados, as informações necessárias fornecidas pelo usuário, são: custo de produção, custo de estoque, multa por atraso de entrega, quantidade de períodos, capacidade e demanda de cada período. Como saída de dados tem-se o planejamento da produção. A capacidade agora deve ser informada em unidade de tempo e não mais em unidade de item para os modos de produção normal e horas extras, isso porque dependendo do tempo necessário para produzir um produto, um recurso pode produzir uma determinada quantidade de um produto e uma quantidade diferente de outro produto. E para relacionar as famílias de itens às capacidades dos recursos, e à quais recursos são necessários para produzir uma unidade de produto daquela família, criou-se a matriz de uso, conforme apresentado no capítulo anterior, uma matriz para cada recurso.

\subsection{UM EXEMPLO}

O exemplo que será apresentado a seguir tem o objetivo de mostrar o funcionamento do aplicativo, mas problemas de maior porte podem ser executados. $\mathrm{O}$ modelo também foi utilizado em uma empresa do segmento de alimentos com sucesso.

Seja um planejamento para 3 períodos, 2 famílias de itens e 2 recursos. Os itens podem ser estocados, terceirizados e entregues ao cliente com atraso. As demandas e capacidades para os períodos são apresentadas nos QUADROS 6 e 7, respectivamente.

\begin{tabular}{|c|c|c|c|c|c|c|}
\hline Período & \multicolumn{2}{|c|}{1} & \multicolumn{2}{c|}{2} & \multicolumn{2}{|c|}{3} \\
\hline Item & 1 & 2 & 1 & 2 & 1 & 2 \\
\hline Demanda & 100 & 50 & 400 & 80 & 300 & 100 \\
\hline
\end{tabular}

FONTE: Os autores (2015).

Os custos de produção são apresentados no QUADRO 8. O valor de 100 u.m. usado no QUADRO 8 significa que não é possível terceirizar no $1^{\circ}$ período de planejamento. 


\begin{tabular}{|c|c|c|c|}
\hline Período & Modo & $\begin{array}{c}\text { Capacidade } \\
\text { Recurso 1 }\end{array}$ & $\begin{array}{c}\text { Capacidade } \\
\text { Recurso 2 }\end{array}$ \\
\hline \multirow{2}{*}{$\mathrm{i}=1,2,3$} & $\begin{array}{c}\text { Normal } \\
\text { (em unidades de tempo) }\end{array}$ & 44 & 44 \\
\cline { 2 - 4 } & $\begin{array}{c}\text { Hora extra } \\
\text { (em unidades de tempo) }\end{array}$ & 10 & 10 \\
\cline { 2 - 4 } & $\begin{array}{c}\text { Terceiro } \\
\text { (em unidades de item) }\end{array}$ & 120 & 120 \\
\hline
\end{tabular}

QUADRO 7 - Capacidade

FONTE: Os autores (2015).

Os custos de estoque por modo de produção e período em u.m. é de 0,2/período para o item 1 e 0,4/período para o item 2, independente do modo de produção. Os custos de atraso por modo de produção e período em u.m. considerados foram: 0,3/período por unidade do item 1 e 0,5/período para o item 2.

\begin{tabular}{|c|c|c|c|c|c|c|c|}
\hline & $\begin{array}{c}\text { Período de } \\
\text { entrega }\end{array}$ & \multicolumn{2}{|c|}{1} & \multicolumn{2}{|c}{2} & \multicolumn{2}{c|}{3} \\
\hline $\begin{array}{c}\text { Período de } \\
\text { produção }\end{array}$ & Modo Item & 1 & 2 & 1 & 2 & 1 & 2 \\
\hline \multirow{3}{*}{1} & normal & 2 & 4 & 2 & 4 & 2 & 4 \\
\cline { 2 - 9 } & hora extra & 4 & 6 & 4 & 6 & 4 & 6 \\
\cline { 2 - 9 }$y$ & terceiro & 100 & 100 & 100 & 100 & 100 & 100 \\
\hline \multirow{3}{*}{2} & normal & 2 & 4 & 2 & 4 & 2 & 4 \\
\cline { 2 - 9 } & hora extra & 4 & 6 & 4 & 6 & 4 & 6 \\
\cline { 2 - 9 } & terceiro & 6 & 8 & 6 & 8 & 6 & 8 \\
\cline { 2 - 9 } & normal & 2 & 4 & 2 & 4 & 2 & 4 \\
\cline { 2 - 9 } & hora extra & 4 & 6 & 4 & 6 & 4 & 6 \\
\hline
\end{tabular}

QUADRO 8 - Custos de produção por modo de produção e período em u.m.

FONTE: Os autores (2015).

A utilização dos recursos 1 e 2 estão representados nos QUADROS 9 e 10, respectivamente. A produção do item 1 utiliza 0,2 unidades do recurso 1 , se for no modo normal ou em hora extra. No modo terceirizado como a capacidade disponível está em unidades de itens que a empresa terceirizada pode se comprometer a produzir, o uso do recurso é de 1 unidade por unidade demandada. Da mesma forma analisa-se o recurso 2.

\begin{tabular}{|c|c|c|c|}
\hline & $\begin{array}{c}\text { Período de } \\
\text { entrega }\end{array}$ & \multicolumn{2}{|c|}{$\mathrm{i}=1,2,3$} \\
\hline $\begin{array}{c}\text { Período de } \\
\text { produção }\end{array}$ & Modo & 1 & 2 \\
\hline \multirow{3}{*}{$\mathrm{i}=1,2,3$} & normal & 0,2 & 0,5 \\
\cline { 2 - 4 } & hora extra & 0,2 & 0,5 \\
\cline { 2 - 4 } & terceiro & 1 & 1 \\
\hline
\end{tabular}

QUADRO 9 - Uso do recurso 1 


\begin{tabular}{|c|c|c|c|}
\hline & $\begin{array}{c}\text { Período de } \\
\text { entrega }\end{array}$ & \multicolumn{2}{|c|}{$\mathrm{i}=1,2,3$} \\
\hline $\begin{array}{c}\text { Período de } \\
\text { produção }\end{array}$ & Modo Item & 1 & 2 \\
\hline \multirow{3}{*}{$\mathrm{i}=1,2,3$} & normal & 0,15 & 0,3 \\
\cline { 2 - 4 } & hora extra & 0,15 & 0,3 \\
\cline { 2 - 4 } & terceiro & 1 & 1 \\
\hline
\end{tabular}

QUADRO 10- Uso do recurso 2

O resultado do planejamento está apresentado no QUADRO 11. Em nenhum dos períodos a capacidade normal foi suficiente para atender a demanda de qualquer item, mas com as horas extras foi possível atender a demanda do item 1 no $1^{\circ}$ período. No $2^{\circ} \mathrm{e}$ $3^{\circ}$ períodos a demanda do item 1 foi atendida com estoque. Quanto ao item 2, houve atraso na entrega do pedido do $1^{\circ}$ período além de que a produção teve que ser terceirizada em todos os períodos. Essa situação pode ser interpretada da seguinte forma: se essa demanda alta acontece frequentemente, deve-se pensar em aumentar a capacidade da empresa, ou manter a terceirização dos produtos, comparando os custos e verificando qual das situações vale a pena, considerando a aquisição de novos recursos e mão de obra. Outra opção pode ser verificar qual o recurso gargalo e se é possível aumentar a capacidade, ou então não aceitar tantos pedidos para esse período. Esta é a função deste modelo de planejamento, discutir o impacto dos custos, da necessidade de ampliação de capacidade ou contratar terceiros, entre outros.

\begin{tabular}{|c|c|c|c|c|c|c|c|}
\hline & Período de & \multicolumn{2}{|c|}{1} & \multicolumn{2}{|c|}{2} & \multicolumn{2}{|c|}{3} \\
\hline $\begin{array}{l}\text { Período de } \\
\text { produção }\end{array}$ & Modo Item & 1 & 2 & 1 & 2 & 1 & 2 \\
\hline \multirow{3}{*}{1} & normal & 60 & 0 & 160 & 0 & 0 & 0 \\
\hline & hora extra & 40 & 4 & 0 & 0 & 0 & 0 \\
\hline & terceiro & 0 & 0 & 0 & 0 & 0 & 0 \\
\hline \multirow{3}{*}{2} & normal & 0 & 0 & 190 & 0 & 30 & 0 \\
\hline & hora extra & 0 & 0 & 50 & 0 & 0 & 0 \\
\hline & terceiro & 0 & 46 & 0 & 74 & 0 & 0 \\
\hline \multirow{3}{*}{3} & normal & 0 & 0 & 0 & 0 & 220 & 0 \\
\hline & hora extra & 0 & 0 & 0 & 0 & 50 & 0 \\
\hline & terceiro & 0 & 0 & 0 & 6 & 0 & 100 \\
\hline
\end{tabular}

QUADRO 11- Resultado do Planejamento

FONTE: Os autores (2015).

\section{CONCLUSÕES FINAIS}

Durante o desenvolvimento desse trabalho o modelo do transporte foi adaptado para o planejamento da produção. A adaptação para apenas um tipo de item e com um recurso foi simples, encontrada inclusive na bibliografia pesquisada tal como em [2] do trabalho de Eregunc e Tufekci. A ampliação do modelo para vários itens e vários recursos gerou o modelo II, e incluiu a criação de uma nova matriz, chamada de matriz de uso, e de novos vetores de capacidade, além de incluir novas colunas na matriz de 
custos e decisão, em consequência do planejamento para várias famílias de itens. Um aplicativo foi desenvolvido para resolver o modelo. $\mathrm{O}$ modelo proposto permite trabalhar com um horizonte de planejamento, famílias de itens e recursos, custos diferenciados por período e modo de produção, possibilita ao usuário escolher se quer ou não terceirizar, estocar e/ou atrasar pedidos. Para as duas últimas situações se escolher a opção de estocar e/ou atrasar, pode determinar por quantos períodos. Com todas essas características o modelo possibilita o gerenciamento da produção, porque fornece informações confiáveis ao usuário e/ou gestor, mostrando quais as variações no comportamento do planejamento no caso de alterações de capacidade, demanda, políticas de terceirização, estoque e atrasos.

\section{REFERÊNCIAS BIBLIOGRÁFICAS}

[1] ARENALES, M.; ARMENTANO, V.; MORABITO, R.; YANASSE, H. Pesquisa Operacional. Rio de Janeiro: Elsevier, 2007.

[2] EREGUNC, S. S.; TUFEKCI, S. A transportation type aggregate production model with bounds on inventory and backordering. European Journal of Operational Research, 35: 414-425, 1988.

[3] GAITHER, N.; FRAZIER, G. Administração de produção e operações. 8. ed. São Paulo: Pioneira Thomson Learning, 2002.

[4] PIZZOLATO, A. L.; GANDOLPHO,A.A. Técnicas de Otimização. Rio de Janeiro, LTC, 2012. 